Subscriber access provided by READING UNIV

Article

\title{
The Role of Gold Adatoms in the Adsorption of Sulfide Species on the Gold(001)-Hex Surface
}

Flavia Lobo Maza, Pilar Carro, Carolina Vericat, Klaus Kern, Roberto C. Salvarezza, and Doris Grumelli J. Phys. Chem. C, Just Accepted Manuscript • DOI: 10.1021/acs.jpcc.7b11059 • Publication Date (Web): 29 Dec 2017

Downloaded from http://pubs.acs.org on December 30, 2017

\section{Just Accepted}

"Just Accepted" manuscripts have been peer-reviewed and accepted for publication. They are posted online prior to technical editing, formatting for publication and author proofing. The American Chemical Society provides "Just Accepted" as a free service to the research community to expedite the dissemination of scientific material as soon as possible after acceptance. "Just Accepted" manuscripts appear in full in PDF format accompanied by an HTML abstract. "Just Accepted" manuscripts have been fully peer reviewed, but should not be considered the official version of record. They are accessible to all readers and citable by the Digital Object Identifier (DOI®). "Just Accepted" is an optional service offered to authors. Therefore, the "Just Accepted" Web site may not include all articles that will be published in the journal. After a manuscript is technically edited and formatted, it will be removed from the "Just Accepted" Web site and published as an ASAP article. Note that technical editing may introduce minor changes to the manuscript text and/or graphics which could affect content, and all legal disclaimers and ethical guidelines that apply to the journal pertain. ACS cannot be held responsible for errors or consequences arising from the use of information contained in these "Just Accepted" manuscripts. 


\title{
The Role of Gold Adatoms in the Adsorption of Sulfide Species on the Gold(001)-hex Surface
}

\author{
Flavia Lobo Maza ${ }^{1}$, Pilar Carro ${ }^{2}$, Carolina Vericat ${ }^{1}$, Klaus Kern ${ }^{3,4}$, Roberto C. Salvarezza ${ }^{1}$ and Doris \\ Grumelli*1 \\ ${ }^{1}$ Instituto de Investigaciones Fisicoquímicas Teóricas y Aplicadas (INIFTA), Facultad de Ciencias \\ Exactas, Universidad Nacional de La Plata - CONICET-Sucursal 4 Casilla de Correo 16, (1900) La \\ Plata, Argentina \\ ${ }^{2}$ Área de Química Física, Departamento de Química, Facultad de Ciencias, Universidad de La Laguna, \\ Instituto de Materiales y Nanotecnología, Avda. Francisco Sánchez, s/n 38200-La Laguna, Tenerife, \\ Spain \\ ${ }^{3}$ Max Planck Institute for Solid State Research, Heisenbergstrasse 1, D-70569 Stuttgart, Germany \\ ${ }^{4}$ Institut de Physique, Ecole Polytechnique Fédéerale de Lausanne, 1015 Lausanne, Switzerland \\ *Corresponding author: Doris Grumelli \\ email: doris@inifta.unlp.edu.ar
}




\begin{abstract}
We have studied the adsorption of reduced sulfur species on the $\mathrm{Au}(001)$-hex surface from diluted ethanolic sulfide solutions by STM, XPS, electrochemical techniques and DFT calculations. Our results reveal the absence of $\mathrm{S}$ multilayers because all $\mathrm{S}$ species are bonded to Au surface atoms. Monomeric $\mathrm{S}$ adsorbs at hollow sites forming a $(2 \times 2)$ lattice, while $S_{2}$ species either capture the gold adatoms $\left(\mathrm{Au}_{\mathrm{ad}}\right)$ that result from the hex lifting or remove $\mathrm{Au}_{\mathrm{ad}}$ from the substrate forming well-ordered $\mathrm{S}_{8}-$ like structures. These are formed by four $\mathrm{S}_{2}$ species coordinated by a central $\mathrm{Au}_{\mathrm{ad}}$, with AuSS interatomic distances closely resembling those of a metal disulfide. Our results explain not only the predominant surface structures on the gold surface but also topographic features, like $\mathrm{Au}_{\mathrm{ad}}$ islands and $\mathrm{Au}$ vacancy islands, and also the chemical nature of adsorbed species from XPS S 2p spectra. In contrast to the S heads of small aromatic thiols, which exhibit a low affinity for $\mathrm{Au}_{\mathrm{ad}}$, strongly reactive sulfide species react with the metal adatoms leading to well-ordered complexes.
\end{abstract}




\section{Introduction}

The adsorption of $\mathrm{S}$ on gold has attracted significant interest not only due to the fact that small gold nanoparticles on oxides catalyze certain reactions both in gas and liquid phase and of the general poisoning effect of sulfur in heterogeneous catalysts ${ }^{12-3}$ but also because of the possible application of sulfur-covered nanoparticles in medicine ${ }^{4}$ and chemical sensors. ${ }^{5}$ Most of the present information is related to the (111) face, although nanoparticles and nanostructured materials also exhibit an important proportion of $\{100\}$ planes. $^{6}$

It is well known that $\mathrm{S}$ arranges in different structures on $\mathrm{Au}(111)$ according to its surface coverage, regardless of the phase (gas or solution) from which adsorption takes place. In general, it is accepted that S adsorption lifts the $22 \times \sqrt{ } 3$ "herringbone" reconstruction, forming organized rows of $S$ adatoms. ${ }^{7}$ Upon further adsorption a diluted $(5 \times 5)$ structure is formed at coverage $(\theta) \sim 0.25 \mathrm{ML}^{8}$, which has been identified by means of low-energy electron diffraction (LEED). This phase then transforms into the denser $(\sqrt{3} \times \sqrt{ } 3)$ R30 with $\theta=0.33$ which, according to theoretical calculations, could coexist with quasi-linear chains. ${ }^{9}$ Finally, by adding more S, the monomeric S atoms on the Au surface condense forming polysulfides that organize in different shapes: trimers, tetramers, and more frequently, octomerlike arrangements with $\theta=0.66 .^{10}$ These polysulfide patterns are observed when $\mathrm{Au}(111)$ substrates are exposed either to $\mathrm{S}_{2}{ }^{8}, \mathrm{SH}_{2}{ }^{11}, \mathrm{SO}_{2}{ }^{12}$ vapors, or immersed in solutions containing $\mathrm{Na}_{2} \mathrm{~S}^{10}$ or organic molecules that could act as sulfur precursors and coexist with a large number of gold vacancy islands $(\theta \approx 0.22){ }^{13-14} 15-16$

In contrast to the well documented knowledge of $\mathrm{S}$ adsorption on the $\mathrm{Au}(111)$ face, adsorption data on the $\mathrm{Au}(001)$ face are scarce. Recently, it has been observed that the adsorption of $S_{2}$ species from the gas phase induces the lifting of the $(5 \times 20)$ hex reconstruction leading to the $(1 \times 1)$ surface. ${ }^{17}$ It has been also reported that $S_{2}$ initially dissociates to yield monomeric sulfur and a $S(2 \times 2)$ phase with $\theta=0.25$ is 
formed on $\mathrm{Au}(001)-(1 \times 1)$. The addition of more $\mathrm{S}_{2}$ results first in the formation of trimers $\left(\mathrm{S}_{3}\right)$ around the $S$ atoms of the $(2 \times 2)$ phase, and finally in a $c(2 \times 4)$ lattice consisting of $S_{2}$ species on top of the $S_{3}$ lattice. ${ }^{17}$ On the other hand, when piperazine-dithiocarbamate was used as a precursor for the spontaneous deposition of sulfur on the $\mathrm{Au}(001)$ surface in alkaline solution, following the lifting of the hex-reconstruction and island formation, the diluted $\mathrm{S}$ phase organizes into a quasi-square lattice with nearest neighbor distances $0.45 \pm 0.01 \mathrm{~nm} .{ }^{18}$ This structure was assigned to a $(\sqrt{ } 2 \times \sqrt{ } 2) \mathrm{S}$ lattice residing on an expanded first layer of $\mathrm{Au}$ atoms with $0.325 \mathrm{~nm}$ interatomic distance. The system then evolves at higher coverage forming $\mathrm{S}_{4}, \mathrm{~S}_{6}$ and the well-known rectangular patterns formed by $8 \mathrm{~S}$ atoms on top of the diluted S lattice. In contrast to the adsorption of S species and S-containing molecules on Au(111), which has been said to induce gold sulfide formation ${ }^{12,17,18}$, Au atom removal from the substrate ${ }^{19}$ and gold adatom $\left(\mathrm{Au}_{\mathrm{ad}}\right)$-molecule complex formation, ${ }^{19,20}$ the possible interaction of adsorbed $\mathrm{S}$ atoms with $\mathrm{Au}$ atoms (resulting either from the hex lifting or from $\mathrm{Au}$ atom surface removal) has not been considered in previous works. The exception is a recent study of sulfide adsorption on the $\mathrm{Au}(001)$ surface $^{21}$ by STM under electrochemical control where, by analogy with previous reports ${ }^{12}$, the $8 \mathrm{~S}$ atoms organized in square-like ("ring-like") structures were interpreted as a gold sulfide.

The $\mathrm{Au}(001)$-hex surface is an interesting environment to test the interactions between $\mathrm{S}$ and $\mathrm{S}$ containing molecules with $\mathrm{Au}_{\mathrm{ad}}$. In fact, the $(5 \times 20)$ hex reconstruction, hereafter denoted as $\mathrm{Au}(001)$ hex surface, has an excess of $25 \%$ of surface atoms that, upon electrochemical lifting, generates $\mathrm{Au}_{\mathrm{ad}}$ that mostly adsorb at step edges/kink sites or nucleate and grow to yield gold islands. ${ }^{22}$ However, in the case of adsorbate induced lifting the $\mathrm{Au}_{\mathrm{ad}}$ can end not only at low coordinated surface sites or as gold islands, but also could react directly with the adsorbates, forming different species. We have recently shown that when small aromatic thiols, such as 6-mercaptopurine, ${ }^{23}$ lift the reconstruction the ejected adatoms do not interact with thiol molecules to form thiol- $\mathrm{Au}_{\mathrm{ad}}$ complexes. Instead, molecules prefer to 
form adatom-free well-ordered lattices, while $\mathrm{Au}_{\mathrm{ad}}$ form small irregular islands, i.e. the S-heads of these thiol molecules have a low affinity for $\mathrm{Au}_{\mathrm{ad}}$. In this context, it is interesting to test the ability of the simplest (and highly reactive) S species to react with a large amount of freshly produced Au adatoms. Thus, in order to reveal the fate of the $\mathrm{Au}_{\mathrm{ad}}$ in a S-rich environment, we have adsorbed $\mathrm{S}$ species on the $\mathrm{Au}(001)$-hex surface from diluted ethanolic solutions using short immersion times to avoid $\mathrm{S}$ multilayer formation.

\section{Experimental Methods}

\section{Sample preparation}

$\mathrm{Au}(001)$ single-crystal substrates (MaTeck) were cleaned by repeated cycles of sputtering with $\mathrm{Ar}^{+}$ions and annealing at $825 \mathrm{~K}$ under ultra-high vacuum (UHV) leading to the $\mathrm{Au}(001)$-(hex) surface reconstruction, ${ }^{22}$ which has been confirmed by LEED and STM. This surface is characterized by stripes $1.45 \mathrm{~nm}$ wide separated by $0.07 \mathrm{~nm}$ deep channels. S adsorption (Aldrich, 98\%) was made by immersion of the $\mathrm{Au}(001)$-hex surfaces in $50 \mu \mathrm{M} \mathrm{Na} \mathrm{Na}_{2} \mathrm{~S}$ ethanolic solutions (BASF 99\%) for $10 \mathrm{~min}$. Other immersion times and concentrations were studied using both ethanol and water as solvents with similar results, but with concomitant $\mathrm{S}$ multilayer formation that complicated data interpretation. Then samples were removed from the solution, rinsed with ethanol and dried under $\mathrm{N}_{2}$ before they were reintroduced into UHV.

\section{STM imaging}

Samples were imaged in a home-made UHV STM operating at room temperature. W tips were used with $100-600 \mathrm{mV}$ tunneling voltages and 100-350 pA tunneling currents. The STM was calibrated in $\mathrm{x}$, $\mathrm{y}$ and $\mathrm{z}$ directions using the stripes of the well-known $\mathrm{Au}(001)$-hex surface reconstruction. WsXM software was used for image analysis. ${ }^{24}$ 


\section{Electrochemical experiments}

A home-built sample transfer system between UHV and electrochemical environment was used. After preparation or characterization the samples were brought to the transfer chamber and argon atmosphere (5.0 purity, $p=1$ bar) was established.

Cyclic voltammetry was made with an Autolab PGSTAT30 potentiostat and a three-electrode conventional electrochemical cell. A large area Pt coil was used as counter electrode and a silver/silver chloride $(3 \mathrm{M} \mathrm{KCl})$ electrode was employed as reference electrode $(\mathrm{RE})$. All the potentials in the text are referred to that RE. Aqueous $0.1 \mathrm{M} \mathrm{NaOH}$ solutions were prepared by using $\mathrm{NaOH}$ pellets (SigmaAldrich; $99.99 \%$ trace metals basis) and ultrapure water with $18.2 \mathrm{M} \Omega \mathrm{cm}$ resistivity (Millipore Products, Bedford).

Sulfur reductive desorption curves were performed at room temperature by scanning the potential from $0.2 \mathrm{~V}$ to $-1.4 \mathrm{~V}$ at $0.05 \mathrm{Vs}^{-1}$ in the $0.1 \mathrm{M} \mathrm{NaOH}$ solution. In each case the charge density (q) involved in the reductive desorption peak was obtained by integration of the peak area. The geometrical area of the single crystal electrode $\left(0.196 \mathrm{~cm}^{2}\right)$ was used to calculate the current densities. This figure was taken as an indication of the surface coverage of the thiol SAM.

\section{XPS}

XPS measurements were performed in a separate UHV system. The freshly prepared S-covered Au substrates were transferred into the UHV system. Photoemission experiments for the S-covered substrates were carried out in a commercial KRATOS AXIS ULTRA system with a monochromatized $\mathrm{Al} \mathrm{K} \alpha$ source with a base pressure in the lower $10^{-10}$ mbar range. The binding energy (BE) scales for the SAMs on Au surfaces were calibrated by setting the $\mathrm{Au} 4 \mathrm{f}_{7 / 2} \mathrm{BE}$ to $84.0 \mathrm{eV}$ with respect to the Fermi level. High-resolution S 2p and Au 4f spectra were acquired with an analyzer pass energy of $20 \mathrm{eV}$. 
Analysis of the XPS data was performed with XPS Peak software. Shirley type backgrounds were subtracted from the spectra before the fitting procedure and peaks that are a combination of Lorenzian and Gaussian functions were employed for all regions. In the case of the $S 2 p$ signal, the spin-orbit doublet separation was fixed at $1.18 \mathrm{eV}$ and an area ratio of 2:1 was used. The coverage from XPS spectra was roughly estimated by comparing the ratio of the normalized intensities with that corresponding to SAMs of well-known coverage whose spectra were taken with the same instrument (6mercaptopurine on $\mathrm{Au}(001)-(1 \mathrm{x} 1)$ and $\mathrm{Au}(001)-\mathrm{hex}){ }^{23}$

\section{Computational Methods}

Density functional calculations were performed with the periodic plane-wave basis set code VASP 5.2.12. ${ }^{25-26}$ The scheme of non-local functional proposed by Dion et al, ${ }^{27} \mathrm{vdW}-\mathrm{DF}$, and the optimized Becke88 exchange functional optB88-vdW ${ }^{28}$ were used to take into account van der Waals (vdW) interactions. The projector augmented plane wave (PAW) method was used to represent the atomic cores using the PBE potential. ${ }^{29}$ The electronic wave functions were expanded in a plane-wave basis set with a $420 \mathrm{eV}$ cutoff energy. Optimal grids of Monkhorst-Pack ${ }^{30}$ k-points $2 \times 9 \times 1$ were used for numerical integration in the reciprocal space of the $(2 \times 2)$ and $(2 \sqrt{ } 2 \times 2 \sqrt{ } 2)-R 45^{\circ}$ unit cells described in the experimental results section. Because S adsorption lifts the hex-reconstruction we have used a Au(001)$(1 \times 1)$ substrate represented by five atomic layers and a vacuum of $\sim 17 \AA$ that separates two successive slabs in our calculation. Surface relaxation is allowed in the three uppermost Au layers of the slab, as well as the atomic coordinates of the adsorbed species, were allowed to relax without further constraints. The atomic positions were relaxed until the force on the unconstrained atoms was $<0.03 \mathrm{eV} \AA^{-1}$. The adsorbates were placed just on one side of the slab and all calculations include a dipole correction. The adsorbed S species, taken as were optimized in an asymmetric box of $20 \AA \times 20 \AA \times 40 \AA$. The 
calculated Au lattice constant is $4.16 \AA$, which compares reasonably well with the experimental value (4.078 $\AA^{31}{ }^{31}$ The average binding energy per adsorbed 6MP species on $\mathrm{Au}(001)-(1 \times 1)$ surfaces, $E_{b}$, is defined in Eq. [1]:

$$
E_{b}=\frac{1}{N_{S}}\left[E^{S / A u}-E^{A u}-N_{S} E^{S}\right]
$$

where, $E^{S / A u}, E^{A u}$ and $E^{S}$ stand for the total energy of the adsorbate-substrate system, the total energy of the Au slab, and the energy of the adsorbed $\mathrm{S}$, respectively, whereas $N_{S}$ is the number of $\mathrm{S}$ atoms in the surface unit cell. A negative number indicates that adsorption is exothermic with respect to the separate clean surface and $\mathrm{S}$ atom. The Gibbs free energy of adsorption of each surface structure $(\gamma)$ was approximated through the total energy from DFT calculations by using equation [2]:

$$
\gamma=\frac{N_{S} E_{b}+E_{r e c}}{A}
$$

where $\mathrm{E}_{\text {rec }}$ is the reconstruction energy per unit cell defined by,

$$
E_{\text {rec }}=E_{A u(001)}^{R}-E_{A u(001)}^{U}-n_{a d} E_{b u l k}^{A u}[3]
$$

where $E_{A u(001)}^{R}$ is the energy of the clean surface of each model, after removing the adsorbate, and $\mathrm{E}_{\mathrm{Au}(001)}^{\mathrm{U}}$ is the energy of the unreconstructed clean substrate. Considering that we are concerned with free energy differences, it is reasonable to assume that the contributions coming from the configurational entropy, the vibrations and the work term $p V$, can be neglected. DFT calculations make systematic (but unknown) errors relative to the true solution of the Schrödinger equation because of the approximate nature of the exchange-correlation functional. However if two DFT calculations compare chemically similar states, then the systematic errors in these two calculations are also similar. In other words, 
relative energies can be calculated accurately. In this sense, we can therefore expect a good level of accuracy in the adsorption energy.

Constant current STM images of the optimized lattices were simulated by using the Tersoff-Hamman method in its most basic form with the STM tip approximated as a point source.(Tersoff, J.; Hamann, D. R., Theory of the Scanning Tunneling Microscope. ${ }^{32}$

\section{Results and Discussion}

A typical STM image taken on the $\mathrm{Au}(001)$-hex substrate after immersion in $10 \mu \mathrm{M}$ ethanolic sodium sulfide solution for $10 \mathrm{~min}$ is shown in Figure 1a. The STM image reveals smooth terraces, with bright islands $\sim 0.20 \mathrm{~nm}$ in height (yellow arrow) separated by steps of the same height (light blue arrow). The cross section analysis along the steps and bright islands (inset in Figure 1a) indicates that they correspond to $\mathrm{Au}$ islands. They result from the lifting of the hex reconstruction to form the $(1 \times 1)$ surface, a process induced by the sulfur adsorption. ${ }^{17}$ A careful inspection also reveals the presence of less contrasted features in the image (white arrows). At a higher resolution (Figure 1b) these features appear as rectangular shaped islands (white arrows) with 0.1-0.14 nm height (inset in Figure 1b). Also, some small dark regions corresponding to Au vacancy islands can be observed in the image (dark blue arrow). In this case the depth is smaller than $0.2 \mathrm{~nm}$ due to tip size effects. 
Figure 1. STM data. (a) STM image showing the Au islands (yellow arrows) and steps (light blue arrows), together with less contrasted features (white arrow). Inset: cross section along a step (left) and an Au island (right). The green line indicates the cross section. (b) STM image showing the Au islands (yellow), rectangular $S$ islands (white arrows), vacancy islands (dark blue arrow), and steps (light blue arrow). Inset: cross section of the rectangular $S$ islands. The green line indicates the cross section

High resolution STM images (Figure 2a) reveal that the rectangular islands shown in Figure $1 \mathrm{~b}$ are formed by square-like shaped structures $\sim 0.5 \mathrm{~nm}$ in side length. Also, a square lattice of less bright spots intersecting the square shaped structures at a $45^{\circ}$ angle can be clearly observed in the STM image. At higher resolution the spots exhibit interatomic distances $d=0.58 \pm 0.03 \mathrm{~nm}$ (Figure $2 \mathrm{~b}$ ), a figure consistent with the $(2 \times 2)$ lattice of $S$ atoms already reported for $S$ adsorption on the same substrate from 
the $S_{2}$ gas phase ${ }^{17}$ and electrolyte solutions. ${ }^{21}$ Also, there are other structures coexiting with the squarelike structures, such as zig-zag chains and dimers (Figure 2c). The height difference between S atoms in the ( $2 \times 2)$ lattice (left hand side) and those in square-shaped structures (right hand side) is $\Delta \mathrm{h}=0.06 \pm 0.03$ $\mathrm{nm}$, as shown in Figure 2d, explaining the difference in contrast in the STM images (Figure 2b). Note that this height difference is too small to assign the square-shaped structures to Au islands, i.e. they correspond to adsorbed $\mathrm{S}$ on the gold surface. The inner structure of the square-shaped patterns is difficult to resolve in the raw images (Figure 2b). However, the Fourier analysis clearly shows that these structures are formed by $8 \mathrm{~S}$ atoms (outer spots in Figure 2e left), and the Fourier filtered image (Figure 2e right) reveals four high density nodes. Similar structures have been reported for S adsorption on this face with average nearest neighbor distance $\sim 0.3 \mathrm{~nm} .{ }^{16}$ 

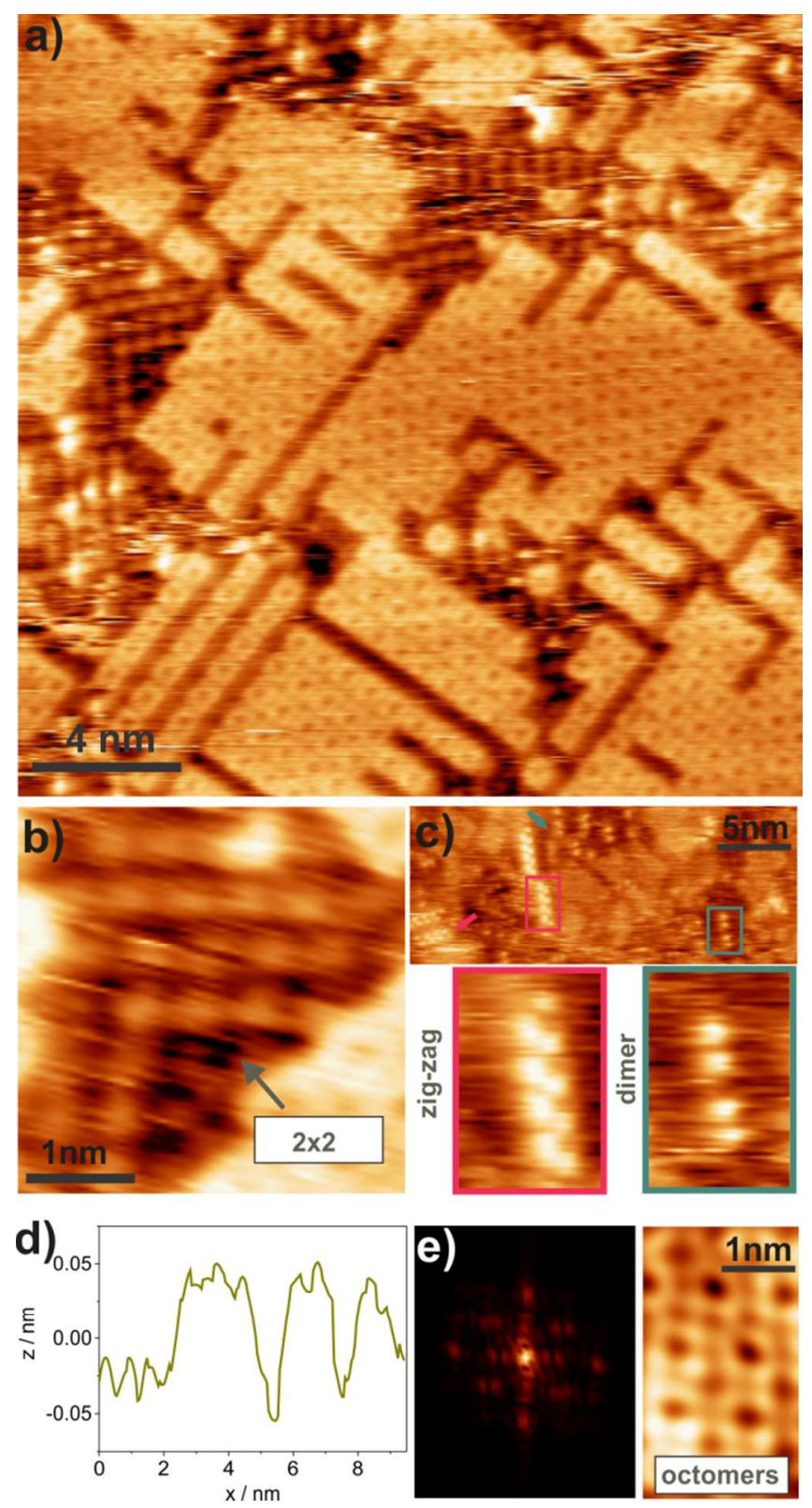

Figure 2. (a) High resolution STM image showing the two different sulfur structures: individual spots and square-like structures. (b) High resolution STM image of the $(2 \times 2)$ lattice. (c) High resolution STM image showing zig-zag chains (pink) and dimers (green). (d) Cross section analysis along $(2 \times 2)$ (left)-square-like structures (right). (e) Fourier transform (left) showing the 8S squarelike structures, and image after Fourier filtering.

A typical XPS spectrum for the $S 2 p$ region after sulfide adsorption on a clean $\mathrm{Au}(001)$ surface is shown in Figure 3a. In principle the peak can be fitted to several doublets, each of them comprised of one pair of Gaussian-Lorentzian functions with spin-orbit splitting $1.2 \mathrm{eV}$ and branching ratio 0.5 . In our case the 
S 2p spectra can be fitted with three components at $161.3 \mathrm{eV}$ (55\% of the total peak area), $162 \mathrm{eV}$ (34\%) and $163.5 \mathrm{eV}(11 \%)$ that have been assigned to adsorbed monomeric $\mathrm{S}\left(\mathrm{S}_{\mathrm{mon}}\right)$, adsorbed polysulfide species $\left(\mathrm{S}_{\text {poly }}\right)$, and $\mathrm{S}$ species non bonded to the Au substrate $\left(\mathrm{S}_{\text {free }}\right)$, respectively. ${ }^{33,17}$ Similar results and interpretation have been made for $\mathrm{S}$ adlayers on $\mathrm{Au}(111)$ prepared from sodium sulfide solutions. ${ }^{34}$ In contrast to $\mathrm{S}_{2}$ adsorption from gas phase, no additional components are needed to fit the spectra of $\mathrm{S}$ on $\mathrm{Au}(001)$, as there is no evidence of elemental $\mathrm{S}(164 \mathrm{eV})$ in the spectra. Also, there is no trace of oxidized gold species in the Au 4f XPS spectra, as they can be fitted with a single doublet that corresponds to metallic gold (Figure 3b). Finally, the estimated S/Au signal ratio results in a total $\mathrm{S}$ coverage $\sim 0.32$, i.e. the corresponding coverage results $\theta_{\text {Smon }} \sim 0.18, \theta_{\text {Spoly }} \sim 0.11, \theta_{\text {Sfree }} \sim 0.03$. In a previous work $^{14}$ it has been considered that the bright square shaped patterns correspond to polysulfides adsorbed on a first layer of diluted monomeric sulfur. However, the proportion of free sulfur species (S not bonded to the Au substrate) in the XPS spectra (Figure 3a, green component) is too small compared to the total amount of sulfur present on the surface. Therefore, the presence of significant amounts of S species adsorbed on top of a first $\mathrm{S}$ adlayer can be discarded. 

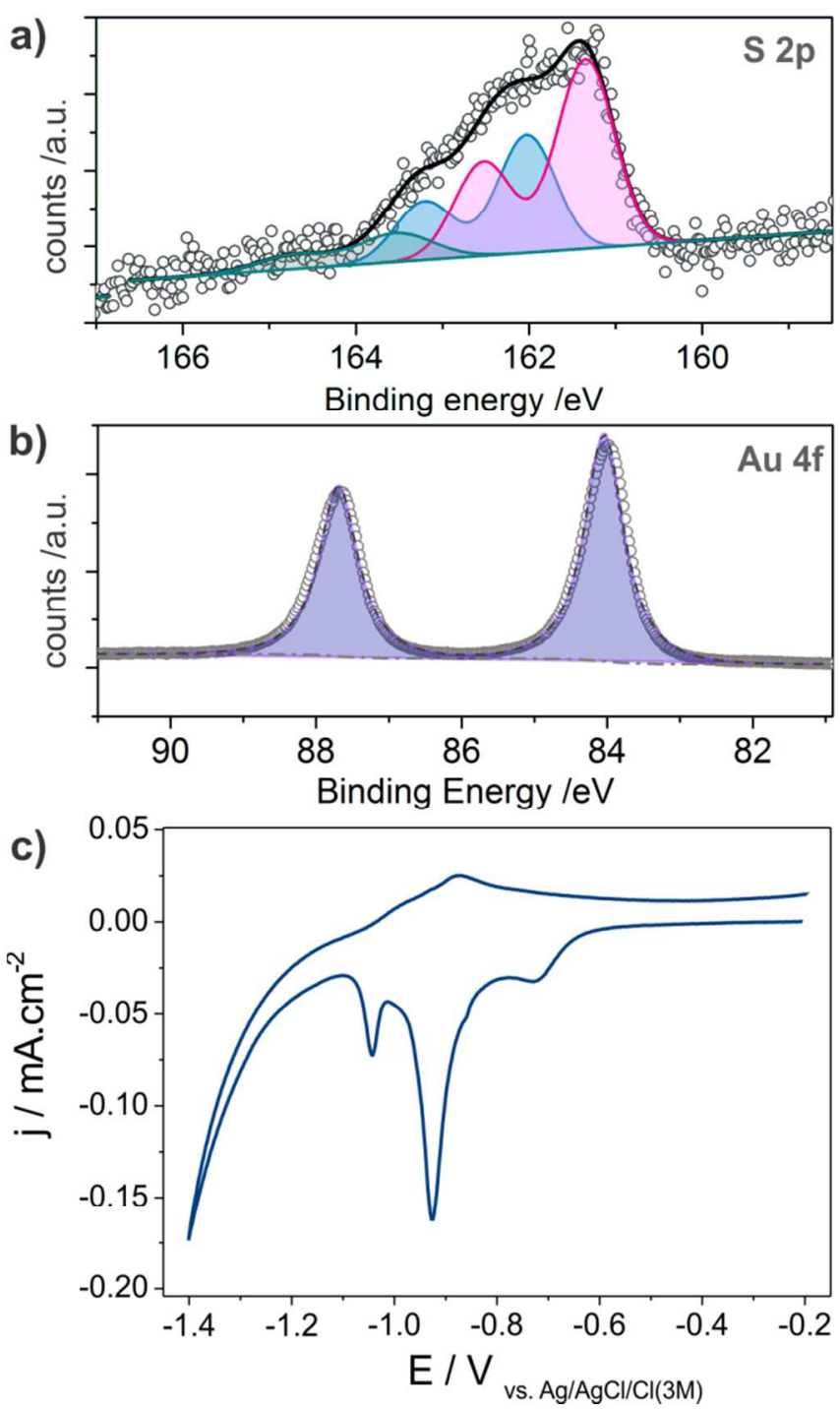

Figure 3. $S$ adsorbed on the Au(001) surface. (a) XPS $S 2 p$ region where the three components are indicated. Red: monomeric $S$ ( $\left.S_{m o n}\right)$; blue: adsorbed polysulfide species $\left(S_{\text {poly }}\right)$; green: unbounded $S$ species $\left(S_{\text {free }}\right)$. (b) XPS Au 4 f region showing the doublet corresponding to metallic gold. (c) Reductive desorption curve recorded in $0.1 \mathrm{MNaOH}$ at a scan rate $0.05 \mathrm{~V} \mathrm{~s}^{-1}$. The peaks at $-0.73 \mathrm{~V},-0.93 \mathrm{~V}$, and 1.2 are assigned to the desorption of $S_{\text {poly }}, S_{m o n}$ and $S_{m o n}$ at step edges, respectively.

Following XPS analysis the samples were transferred to the electrochemical cell (see experimental section) to estimate the amount of chemisorbed S species ( $89 \%$ of the total sulfur from Figure $3 a$ ) from the reductive desorption region in the cyclic voltammogram (Figure 3c). Actually, three current peaks can be clearly observed in the cathodic curve related to reductive desorption. The peak at $-0.73 \mathrm{~V}$ can be assigned to desorption of the polysulfide species (which appear at $162 \mathrm{eV}$ in the XPS spectrum), while 
the main peak at $-0.93 \mathrm{~V}$ can be associated with the desorption of the monomeric $\mathrm{S}$ atoms (peak at 161.3 $\mathrm{eV}$ in Figure 3a). On the other hand, the small peak at $-1.2 \mathrm{~V}$ can be related to the desorption of monomeric S from step edges. The large current at more negative potentials corresponds to the hydrogen evolution reaction. Also, some readsorption of the desorbed S species is observed after reversing the potential scan (in the positive direction), as already reported. ${ }^{10}$

The overall charge density involved in the cathodic peaks is $\sim 0.14 \mathrm{mC} \mathrm{cm}^{-2}$. The estimation of surface coverage from integration of the charge involved in the three cathodic peaks is rather difficult due to peak convolution. Anyway, we have roughly estimated $\mathrm{q}=0.100 \mathrm{mC} \mathrm{cm}^{-2}$ for the peaks assigned to $\mathrm{S}_{\mathrm{mon}}$ $(-0.93 \mathrm{~V}$ and $-1.2 \mathrm{~V})$, and thus $\theta_{\mathrm{Smon}} \sim 0.25$ results assuming the reaction

$$
2 \mathrm{e}+\mathrm{S}-\mathrm{Au}+\mathrm{H}_{2} \mathrm{O} \rightarrow \mathrm{HS}^{-}+\mathrm{Au}+\mathrm{OH}^{-}[4]
$$

On the other hand, by integration of the peak at $-0.73 \mathrm{~V}$, assigned to $\mathrm{S}_{\text {poly }}, \mathrm{q}=0.04 \mathrm{mC} \mathrm{cm}^{-2}$ is obtained. Assuming that polysulfide species are mainly adsorbed $\mathrm{S}_{2}$ that desorb by the reaction

$$
2 \mathrm{e}+\mathrm{S}_{2}-\mathrm{Au}+\mathrm{H}_{2} \mathrm{O} \rightarrow \mathrm{HS}^{-}+\mathrm{S}+\mathrm{Au}+\mathrm{OH}^{-}[5]
$$

we have $\theta_{\text {Spoly }} \sim 0.1$, in reasonable agreement with the coverages obtained from the XPS data.

In the next we will propose models for the two relevant surface structures of $S$ on the $\mathrm{Au}(001)$ substrate observed in the STM images by using DFT calculations. STM results clearly show the presence of the $(2 \times 2) \mathrm{S}$ lattice already reported on the $\mathrm{Au}(001)-(1 \times 1)$ face. ${ }^{35}$ On the other hand, the square-shaped patterns exhibit distances that can be accounted for in terms of a $(2 \sqrt{2} \times 2 \sqrt{2}) \mathrm{R} 45^{\circ}$-S surface structure. In this case we have explored two different situations to understand the fate of the Au adatoms arising from the hex lifting (model I) and the presence of Au vacancies (model II) (Figure 1). The optimized structures for the $(2 \times 2)$ and the $(2 \sqrt{ } 2 \times 2 \sqrt{ } 2) \mathrm{R} 45^{\circ}$-S models and their simulated STM images are shown in 
Figure 4. The $(2 \times 2)$ lattice consists of $\mathrm{S}$ atoms placed at hollow sites with $0.58 \mathrm{~nm}$ distances between them and $\theta=0.25$ (Figure 4a1); its corresponding simulated STM image is shown in Figure 4a2.

On the other hand, the optimized $(2 \sqrt{ } 2 \times 2 \sqrt{ } 2)-\mathrm{R} 45^{\circ}-\mathrm{S}$ structure of model I (Figure $\left.4 \mathrm{~b} 1\right)$ was built by initially placing $8 \mathrm{~S}$ atoms in a square arrangement around a central $\mathrm{Au}_{\mathrm{ad}}$ located at the hollow site of the $\mathrm{Au}(001)-(1 \times 1)$ surface, as shown in Figure 4b3. Upon optimization $\mathrm{S}$ atoms rearrange into four $\mathrm{S}_{2}$ species, forming a $8 \mathrm{~S}$ square-like structure with $\sim 0.46 \mathrm{~nm}$ in side length, around the $\mathrm{Au}_{\mathrm{ad}}$ (Figure 4b4). A detailed analysis of the optimized structure shows that the $S$ atoms of the $S_{2}$ species are bound to the substrate at on-top sites, with one of the $\mathrm{S}$ atoms of each $\mathrm{S} 2$ also binds to the central $\mathrm{Au}_{\mathrm{ad}}$ (Figure 4b4). The simulated STM image (Figure 4b2) is in good agreement with the experimental data.

The optimized structure of model II can be also described as a $(2 \sqrt{ } 2 \times 2 \sqrt{ } 2)$ R $45^{\circ}$-S lattice (Figure $\left.4 \mathrm{c} 1\right)$. In this case it results by initially placing $8 \mathrm{~S}$ atoms in a similar arrangement but with the square center over a $\mathrm{Au}$ atom of the topmost $\mathrm{Au}(001)$ surface, i.e. without the central $\mathrm{Au}_{\mathrm{ad}}$ (Figure $\left.4 \mathrm{c} 3\right)$. We found that the initial system (Figure 4c3) spontaneously evolves lifting this central Au substrate atom, thus yielding a similar surface structure to that shown in Figure 4b1 but with a vacancy below the extracted Au atom (Figure 4c1). Similar results are obtained when $8 \mathrm{~S}$ atoms are placed on $\mathrm{Au}(111) .{ }^{34}$ Note that, in contrast to the $8 \mathrm{~S}$ square-like structure shown in Figure $4 \mathrm{~b} 4$, one the $\mathrm{S}$ atoms of each $\mathrm{S}_{2}$ species is on-top of the $\mathrm{Au}$ site but the other is only connected to the $\mathrm{Au}_{\mathrm{ad}}$ (Figure 4c4). The corresponding simulated STM image is shown in Figure 4c2.

The 8 S square-like structures of models I and II exhibit S-S distances of $0.204 \mathrm{~nm}$, typical of disulfide bonds, while the S-Au distances are $0.24 \mathrm{~nm}$ for both the $\mathrm{S}-\mathrm{Au}_{\mathrm{ad}}$ and $\mathrm{S}-\mathrm{Au}$ substrate bonds, respectively. These distances match very well those measured for AuSS species: Au-S 0.235 and S-S 0.205 nm. ${ }^{36}$ Also, it is widely known that sulfur can form tetra-coordinated complexes with gold. ${ }^{37}$ It should be noted 
that we have tested a large number of DFT models for the square shaped $8 \mathrm{~S}$ structures, although only models I and II are consistent with the experimental data.

The structural and energetic parameters for both surface models are shown in Table 1. Large negative binding energies $\left(E_{b}\right)$ and surface free energies $(\gamma)$ can be observed in all cases, i.e. the proposed surface structures are thermodynamically favored. Monomeric $S$ atoms in the $(2 \times 2)$ lattice exhibit larger $E_{b}$ values than those forming the $S_{2}$ species in the $(2 \sqrt{ } 2 \times 2 \sqrt{ } 2)-R 45^{\circ}$ lattices, in agreement with experimental results $^{1}$ and DFT calculations ${ }^{38}$ for $S$ adsorbed on the $\mathrm{Au}(111)$ surface. In fact, it has been shown that $\mathrm{E}_{\mathrm{b}}$ decreases as the $S$ coverage is increased. The larger $\gamma$ values for the $(2 \sqrt{ } 2 \times 2 \sqrt{ } 2)-\mathrm{R} 45^{\circ}$ models in relation to the $(2 \times 2)$ lattice can be explained from their larger atomic density (see equation 11 and STM images in Figure 2). Note that model I, which has a better coordination of S atoms to the substrate, has a slightly better adsorption energy and stability than model II.

In the next, we outline a singular behavior about the chirality of these surface structures. Chirality can be bestowed upon achiral surfaces via the addition of chiral modifiers ${ }^{39}$ and even via the addition of achiral modifiers, like in our case. It can be observed that both surface structures presented in model I (Figure 4-b1) and model II (Figure 4-c1) are chiral around the Au adatom. However, the interesting difference is that, while the initial surface structure of the model I that starts with an adatom inside the S8 complex (Figure 4-b3) could have evolved towards the other enantiomer, the initial structure of model II (figure4-c3), which does not contain an Au adatom, has chosen to evolve during the geometry relaxation towards this particular enantiomer form (figure 4-c1). Therefore, to properly compare we have chosen to present the same enantiomer form for both models I and II.

The Bader analysis yields -0.31 e for $S$ in the $(2 \times 2)$ lattice while it results in -0.13 (model I)/-0.14 (model II) eV and -0.09 e for $\mathrm{S}$ atoms in the $(2 \sqrt{2} \times 2 \sqrt{2})-\mathrm{R} 45^{\circ}$ lattice bonded to the top Au site and the $\mathrm{Au}_{\mathrm{ad}}$, respectively. On the other hand, the $\mathrm{Au}$ surface atoms have $+0.06 \mathrm{e}$ in the $(2 \times 2)$ lattice and 0 e and 
$+0.16 \mathrm{e}$ for the $\mathrm{Au}$ surface atoms and $\mathrm{Au}_{\mathrm{ad}}$ for the $(2 \sqrt{ } 2 \times 2 \sqrt{ } 2)-\mathrm{R} 45^{\circ}$ phase, respectively. Moreover, the core level shift (CLS) analysis for the $(2 \sqrt{2} \times 2 \sqrt{2})-\mathrm{R} 45^{\circ}$ models indicates that the $\mathrm{S}$ atoms of the $\mathrm{S}_{2}$ species binding to the $\mathrm{Au}_{\mathrm{ad}}$ are shifted $\sim 0.6 \mathrm{eV}$ (model I) $/ 0.5 \mathrm{eV}$ (model II) towards larger $\mathrm{BE}$ with respect to the $\mathrm{S}$ atoms in the $(2 \times 2)$ lattice. On other hand, the $\mathrm{S}$ atoms of the dimers on-top of the $\mathrm{Au}$ substrate atoms show a BE shift of $-0.19 \mathrm{eV}$ (model I) and $0 \mathrm{eV}$ (model II) with respect to the $\mathrm{S}$ atoms in the $(2 \times 2)$ lattice. This last result seems to contradict the Bader analysis because we expect that these less negatively charged $S$ atoms should have larger BE than that of monomeric $S$ in the $(2 \times 2)(-0.31 \mathrm{eV})$. Note, however, that the CLS reflects not only the change in the charge transfer to an atom or molecule but also screening effects by the surrounding atoms, molecules or substrate, and redistribution of charge due to bonding and hybridization. ${ }^{40}$

With these data in mind we can interpret the large $161.3 \mathrm{eV} \mathrm{S} 2 \mathrm{p}$ component as having contributions both from $S$ atoms in the $(2 \times 2)$ and $(2 \sqrt{ } 2 \times 2 \sqrt{ } 2)-R 45^{\circ}$ surface structures, while the less intense $162 \mathrm{eV}$ component only stems from $S$ atoms in the $(2 \sqrt{ } 2 \times 2 \sqrt{ } 2)-\mathrm{R} 45^{\circ}$ phase. 

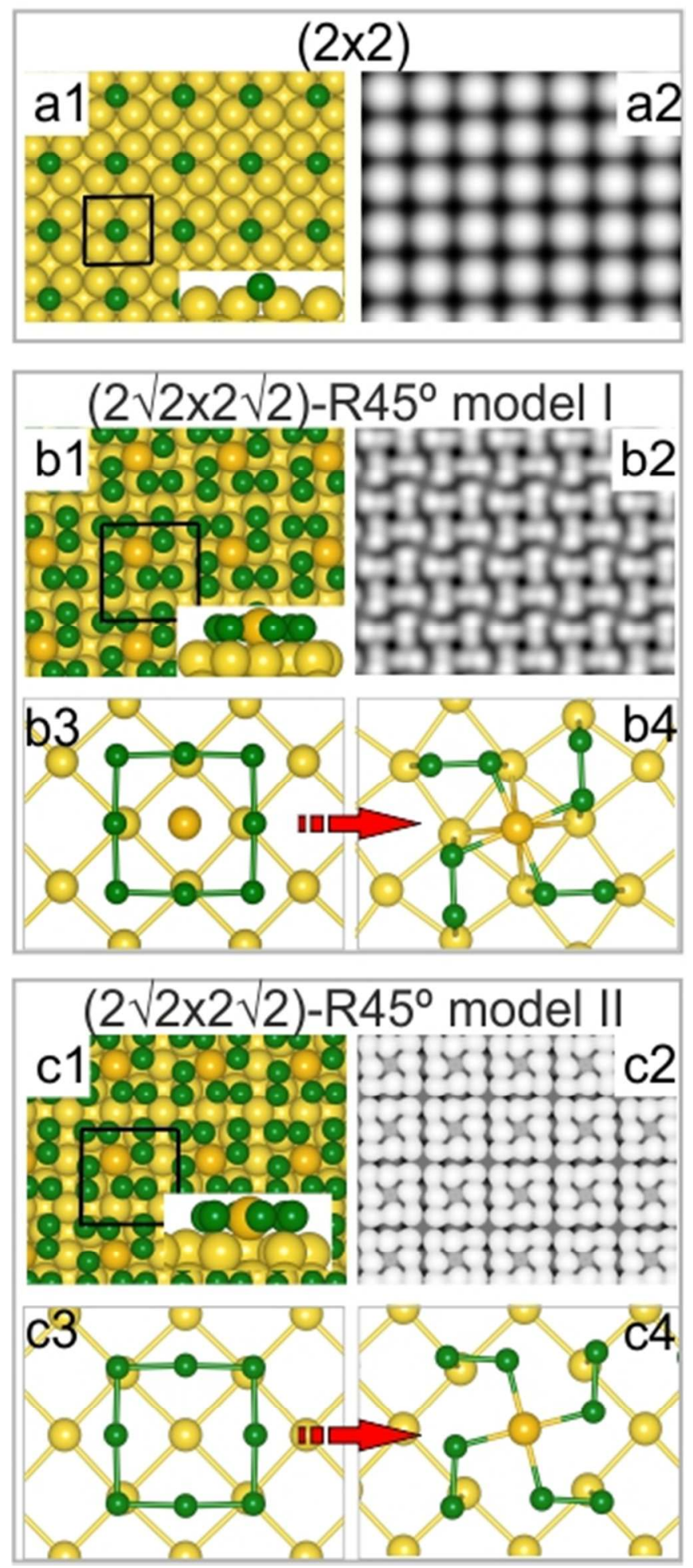

Figure 4. Top and side views (inset) of the optimized surface models for $S$ on $A u(001)-(1 \times 1)$ surface: a1) (2×2); b1) model I for $(2 \sqrt{2} \times 2 \sqrt{2})-\mathrm{R} 45^{\circ}$; c1) model II for $(2 \sqrt{2} \times 2 \sqrt{2})-\mathrm{R} 45^{\circ}$. The corresponding simulated current STM images are depicted in a2), b2) and c2). Initial $8 S$ geometry for models I (b3) 
and II (c3) and final optimized geometry for $(2 \sqrt{2} \times 2 \sqrt{ } 2)-\mathrm{R} 45^{\circ}$ for models I (b4) and II (c4). Yellow: Au atoms; green: $S$ atoms; grey: $C$ atoms; white: $H$ atoms. The corresponding unit cells are indicated in black in al, b1 and cl.

Table 1

\begin{tabular}{|c|c|c|c|}
\hline Substrate & \multicolumn{3}{|c|}{ Au(001)-1x1 } \\
\hline Surface Lattice & $(2 \times 2)$ & $\begin{array}{c}(2 \sqrt{ } 2 \times 2 \sqrt{ } 2)-\mathrm{R} 45^{\circ} \\
\text { Model I }\end{array}$ & $\begin{array}{c}(2 \sqrt{ } 2 \times 2 \sqrt{ } 2)-\mathrm{R} 45^{\circ} \\
\text { Model II }\end{array}$ \\
\hline$\theta_{\mathrm{S}}$ & 0.25 & 1.0 & 1.0 \\
\hline$\theta_{\text {Au_adatom }}$ & 0 & $1 / 8$ & $1 / 8$ \\
\hline$\theta_{\text {vacancy }}$ & 0 & 0 & $1 / 8$ \\
\hline $\boldsymbol{E}_{\boldsymbol{b}} / \mathbf{e V}$ & -4.06 & -3.68 & -3.44 \\
\hline $\boldsymbol{E}_{\text {rec }} / \boldsymbol{e} \boldsymbol{V}$ & - & +0.11 & +0.28 \\
\hline$\gamma / \boldsymbol{m e V} . \AA^{-2}$ & -117.32 & -412.90 & -393.27 \\
\hline
\end{tabular}

We propose that the adsorbed $\mathrm{S}$ atoms accomodate at hollow sites forming the $(2 \times 2)$ lattice, thus optimizing their adsorption energy [reaction 6].

$$
\mathrm{S}_{(\mathrm{ad})}-\mathrm{Au} \rightleftarrows \mathrm{S}_{\mathrm{ad}}-\mathrm{Au}_{\text {hollow site }}[6]
$$

On the other hand, the ejected adatoms resulting from the hex lifting diffuse on the Au surface, and some of them interact forming Au islands [reaction 7]

$$
\mathrm{nAu}_{\mathrm{ad}} \rightarrow\left(\mathrm{Au}_{\mathrm{ad}}\right)_{\mathrm{n}}[7]
$$

Adsorption of additional S atoms leads to the formation of disulfide species adsorbed on the Au surface [reaction 8] 


$$
\mathrm{nS}_{(\mathrm{ad})}-\mathrm{Au} \rightleftarrows \mathrm{n} / 2 \mathrm{~S}_{2(\mathrm{ad})}-\mathrm{Au}[8]
$$

Then, the adsorbed disulfides either react with $\mathrm{Au}_{\mathrm{ad}}$ remaining on the surface [reaction 9]

$$
4 \mathrm{~S}_{2(\mathrm{ad})}-\mathrm{Au}+\mathrm{Au}_{\mathrm{ad}} \rightleftarrows 4 \mathrm{~S}_{2} \mathrm{Au}_{\mathrm{ad}}-\mathrm{Au}[9]
$$

or extract $\mathrm{Au}$ atoms from the terraces [reaction 10]

$$
4 \mathrm{~S}_{2(\mathrm{ad})}-\mathrm{Au} \underset{\mathrm{AS}}{2} \mathrm{Au}_{\mathrm{ad}}-\mathrm{Au}+\mathrm{Au}_{\mathrm{vac}}[10]
$$

resulting in the formation of the $(2 \sqrt{ } 2 \times 2 \sqrt{ } 2)$-R $45^{\circ}$ lattice, which contains $\mathrm{Au}_{\mathrm{ad}}$, while the $\mathrm{Au}$ vacancies produced by [reaction 8] diffuse and nucleate to yield gold vacancy islands [reaction 11]

$$
\mathrm{nAu}_{\mathrm{vac}} \rightarrow\left(\mathrm{Au}_{\mathrm{vac}}\right)_{\mathrm{n}}[11]
$$

Reactions [6-11] account for the different features resulting from sulfide adsorption on the hex surface from solution: the Au islands, the Au vacancy islands, and the $(2 \times 2)$ and $(2 \sqrt{ } 2 \times 2 \sqrt{ } 2))-\mathrm{R} 45^{\circ} \mathrm{S}$ lattices.

\section{Conclusions}

Experimental results from XPS and electrochemistry reveal that $\mathrm{S}$ adsorption lifts the hex reconstruction leading to Au island formation. Under our experimental conditions all the adsorbed $\mathrm{S}$ species are bonded to Au surface atoms. STM and DFT data show that monomeric S adsorbs at hollow sites forming a (2×2) lattice, while $\mathrm{S}_{2}$ species capture $\mathrm{Au}_{\mathrm{ad}}$ resulting from the hex lifting or remove $\mathrm{Au}$ atoms from the substrate, forming well-ordered square-like structures formed by $8 \mathrm{~S}$ atoms. These structures consist of $4 \mathrm{~S}_{2}$ coordinated by a central Au atom, with AuSS interatomic distances that closely resemble a metal disulfide. Our results explain not only the surface structures but also topographic features, such as Au adatom islands and Au vacancy islands. Also, DFT results allow a correct interpretation of the XPS S 2p component in the spectra. Finally, we demonstrate differences in the chemical behavior of adsorbed S species on the $\mathrm{Au}(001)$-hex substrate. While the $\mathrm{S}$ head of small aromatic thiols such as 6- 
mercaptopurine have a low affinity for the $\mathrm{Au}_{\mathrm{ad}}$ resulting from the reconstruction lifting ${ }^{23}$ the simpler and more reactive $\mathrm{S}$ species are able to transform them into well-ordered sulfur-metal complexes.

\section{References}

(1) Rodriguez, J. A.; Dvorak, J.; Jirsak, T.; Liu, G.; Hrbek, J.; Aray, Y.; González, C., Coverage Effects and the Nature of the Metal-Sulfur Bond in S/Au(111): High-Resolution Photoemission and Density-Functional Studies. J. Am. Chem. Soc. 2003, 125, 276-285.

(2) E. Bailie, J.; J. Hutchings, G., Promotion by Sulfur of Gold Catalysts for Crotyl Alcohol Formation from Crotonaldehyde Hydrogenation. Chem. Commun. 1999, 2151-2152.

(3) Min, B. K.; Alemozafar, A. R.; Biener, M. M.; Biener, J.; Friend, C. M., Reaction of Au(111) with Sulfur and Oxygen: Scanning Tunneling Microscopic Study. Top. Catal. 2005, 36, 77-90.

(4) Huergo, M. A.; Maier, C. M.; Castez, M. F.; Vericat, C.; Nedev, S.; Salvarezza, R. C.; Urban, A. S.; Feldmann, J., Optical Nanoparticle Sorting Elucidates Synthesis of Plasmonic Nanotriangles. ACS Nano 2016, 10, 3614-3621.

(5) Fan, Y.; Long, Y. F.; Li, Y. F., A Sensitive Resonance Light Scattering Spectrometry of Trace Hg2+ with Sulfur Ion Modified Gold Nanoparticles. Anal. Chim. Acta 2009, 653, 207-211.

(6) Azcárate, J. C.; Corthey, G.; Pensa, E.; Vericat, C.; Fonticelli, M. H.; Salvarezza, R. C.; Carro, P., Understanding the Surface Chemistry of Thiolate-Protected Metallic Nanoparticles. J. Phys. Chem. Lett. 2013, 4, 3127-3138.

(7) Walen, H.; Liu, D.-J.; Oh, J.; Lim, H.; Evans, J. W.; Kim, Y.; Thiel, P. A., Self-Organization of S Adatoms on $\mathrm{Au}(111): \mathrm{V} 3 \mathrm{r} 30^{\circ}$ Rows at Low Coverage. J. Chem. Phys. 2015, 143, 014704.

(8) Yu, M.; Ascolani, H.; Zampieri, G.; Woodruff, D. P.; Satterley, C. J.; Jones, R. G.; Dhanak, V. R., The Structure of Atomic Sulfur Phases on Au(111). J. Phys. Chem. C 2007, 111, 10904-10914.

(9) Gomez-Carrillo, S. C.; Bolcatto, P. G., Coexistence of [Surd Radical]3 [Times] [Surd Radical]3 and Quasi-Linear Phases of Sulfur Adsorbed ([Capital Theta] = 1/3) on a Gold (111) Substrate. Phys. Chem. Chem. Phys. 2011, 13, 461-466.

(10) Vericat, C.; Andreasen, G.; Vela, M. E.; Salvarezza, R. C., Dynamics of Potential-Dependent Transformations in Sulfur Adlayers on Au(111) Electrodes. J. Phys. Chem. B 2000, 104, 302-307.

(11) McCarley, R. L.; Kim, Y. T.; Bard, A. J., Scanning Tunneling Microscopy and Quartz Crystal Microbalance Studies of Gold Exposed to Sulfide, Thiocyanate, and N-Octadecanethiol. J. Phys. Chem. 1993, 97, 211-215.

(12) Biener, M. M.; Biener, J.; Friend, C. M., Revisiting the S-Au(111) Interaction: Static or Dynamic? Langmuir 2005, 21, 1668-1671.

(13) Koczkur, K. M.; Hamed, E. M.; Houmam, A., Sulfur Multilayer Formation on Au(111): New Insights from the Study of Hexamethyldisilathiane. Langmuir 2011, 27, 12270-12274.

(14) Martínez, J. A.; Valenzuela, J.; Hernandez-Tamargo, C. E.; Cao-Milán, R.; Herrera, J. A.; Díaz, J. A.; Farías, M. H.; Mikosch, H.; Hernández, M. P., Study of Sulfur Adlayers on Au(1 111$)$ from Basic Hydrolysis of Piperazine Bis(Dithiocarbamate) Sodium Salt. Appl. Surf. Sci. 2015, 345, 394-399.

(15) Fischer, J. A.; Zoldan, V. C.; Benitez, G.; Rubert, A. A.; Ramirez, E. A.; Carro, P.; Salvarezza, R. C.; Pasa, A. A.; Vela, M. E., Sulfidization of Au(111) from Thioacetic Acid: An Experimental and Theoretical Study. Langmuir 2012, 28, 15278-15285.

(16) Pensa, E.; Cortés, E.; Corthey, G.; Carro, P.; Vericat, C.; Fonticelli, M. H.; Benítez, G.; Rubert, A. A.; Salvarezza, R. C., The Chemistry of the Sulfur-Gold Interface: In Search of a Unified Model. Accounts of Chemical Research 2012, 45, 1183-1192. 
(17) Jiang, Y.; Liang, X.; Ren, S.; Chen, C.-L.; Fan, L.-J.; Yang, Y.-W.; Tang, J.-M.; Luh, D.-A., The Growth of Sulfur Adlayers on Au(100). J. Chem. Phys. 2015, 142, 064708.

(18) Hernandez-Tamargo, C. E.; Montero-Alejo, A. L.; Pujals, D. C.; Mikosch, H.; Hernández, M. P., Sulfur Dimers Adsorbed on $\mathrm{Au}(111)$ as Building Blocks for Sulfur Octomers Formation: A Density Functional Study. J. Chem. Phys. 2014, 141, 044713.

(19) Guo, Q.; Li, F., Self-Assembled Alkanethiol Monolayers on Gold Surfaces: Resolving the Complex Structure at the Interface by Stm. Phys. Chem. Chem. Phys. 2014, 16, 19074-19090.

(20) Hakkinen, H., The Gold-Sulfur Interface at the Nanoscale. Nat Chem 2012, 4, 443-455.

(21) Schlaup, C.; Wandelt, K., In-Situ Stm Study of Sulfide Adsorption on Au(100) in Alkaline Solution. Surf. Sci. 2015, 631, 165-172.

(22) Grumelli, D.; Cristina, L. J.; Maza, F. L.; Carro, P.; Ferrón, J.; Kern, K.; Salvarezza, R. C., Thiol Adsorption on the $\mathrm{Au}(100)$-Hex and Au(100)-(1 × 1) Surfaces. J. Phys. Chem. C 2015, 119, 14248-14254.

(23) Carro, P.; Müller, K.; Maza, F. L.; Vericat, C.; Starke, U.; Kern, K.; Salvarezza, R. C.; Grumelli, D., 6Mercaptopurine Self-Assembled Monolayers on Gold (001)-Hex: Revealing the Fate of Gold Adatoms. J. Phys. Chem. C 2017, 121, 8938-8943.

(24) Horcas, I.; Fernandez, R.; Gomez-Rodriguez, J. M.; Colchero, J.; Gomez-Herrero, J.; Baro, A. M., Wsxm: A Software for Scanning Probe Microscopy and a Tool for Nanotechnology. Rev. Sci. Instrum. 2007, 78, 013705-8. (25) Kresse, G.; Furthmüller, J., Efficiency of Ab-Initio Total Energy Calculations for Metals and Semiconductors Using a Plane-Wave Basis Set. Comput. Mater. Sci. 1996, 6, 15-50.

(26) Kresse, G.; Hafner, J., Ab Initio. Phys. Rev. B 1993, 48, 13115-13118.

(27) Dion, M.; Rydberg, H.; Schröder, E.; Langreth, D. C.; Lundqvist, B. I., Van Der Waals Density Functional for General Geometries. Phys. Rev. Lett. 2004, 92, 246401.

(28) Jiří, K.; David, R. B.; Angelos, M., Chemical Accuracy for the Van Der Waals Density Functional. J. Phys.: Condens. Matter 2010, 22, 022201.

(29) Blöchl, P. E., Projector Augmented-Wave Method. Phys. Rev. B 1994, 50, 17953-17979.

(30) Monkhorst, H. J.; Pack, J. D., Special Points for Brillouin-Zone Integrations. Phys. Rev. B 1976, 13, 51885192.

(31) Pearson, W. B., In Handbook of Lattice Spacing and Structure of Metals; Pergamon Press, Inc.: New York, 1958.

(32) Tersoff, J.; Hamann, D. R., Theory of the Scanning Tunneling Microscope. Phys. Rev. B 1985, 31, 805-813. (33) Vericat, C.; Vela, M. E.; Andreasen, G.; Salvarezza, R. C.; Vázquez, L.; Martín-Gago, J. A., Sulfur-Substrate Interactions in Spontaneously Formed Sulfur Adlayers on Au(111). Langmuir 2001, 17, 4919-4924.

(34) Lustemberg, P. G.; Vericat, C.; Benitez, G. A.; Vela, M. E.; Tognalli, N.; Fainstein, A.; Martiarena, M. L.; Salvarezza, R. C., Spontaneously Formed Sulfur Adlayers on Gold in Electrolyte Solutions: Adsorbed Sulfur or Gold Sulfide? J. Phys. Chem. C 2008, 112, 11394-11402.

(35) Jiang, Y.; Liang, X.; Ren, S.; Chen, C.-L.; Fan, L.-J.; Yang, Y.-W.; Tang, J.-M.; Luh, D.-A., The Growth of Sulfur Adlayers on $\mathrm{Au}(100), 2015$; Vol. 142, p 064708.

(36) Wang, X.; Liang, B.; Andrews, L., Infrared Spectra and Density Functional Theory Calculations of Coinage Metal Disulfide Molecules and Complexes. Dalton Trans. 2009, 4190-4198.

(37) Bayler, A.; Schier, A.; Schmidbaur, H., Four-Coordinate Gold(I), Silver(I), and Copper(I) Complexes with a Large-Span Chiral Ditertiary Phosphine Ligand. Inorg. Chem. 1998, 37, 4353-4359.

(38) Lustemberg, P. G.; Martiarena, M. L.; Martínez, A. E.; Busnengo, H. F., The Reaction Pathways for Hsch3 Adsorption on $\mathrm{Au}(111)$ : A Density Functional Theory Study. Langmuir 2008, 24, 3274-3279.

(39) Zaera, F., Chirality in Adsorption on Solid Surfaces. Chemical Society Reviews 2017, 46, 7374-7398.

(40) Salvarezza, R. C.; Carro, P., Exploring the Core Level Shift Origin of Sulfur and Thiolates on Pd(111) Surfaces.

Phys. Chem. Chem. Phys. 2015, 17, 24349-24355. 
The Role of Gold Adatoms.

Sulfur adsorption on the Gold(001)-Hex surface

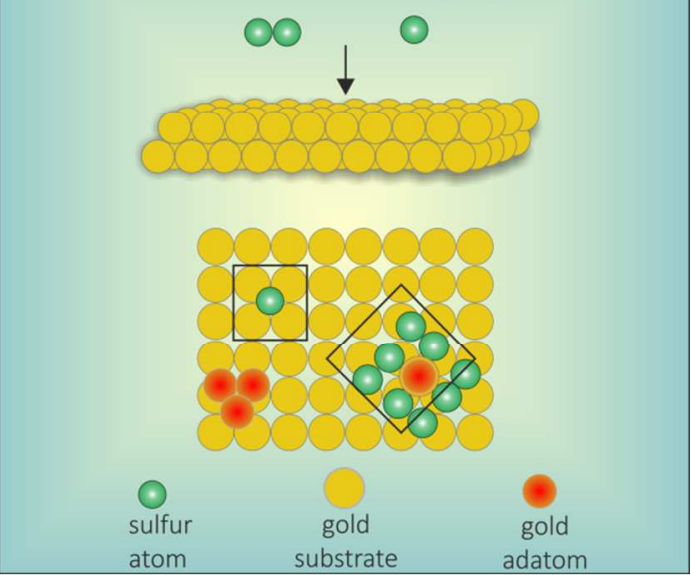

